\title{
Revisiting the Cosmological Principle in a Cellular Framework
}

\author{
L. Zaninetti \\ Dipartimento di Fisica , \\ Via Pietro Giuria 1, \\ 10125 Torino, Italy
}

March 9, 2022

The Cosmological Principle in it's various versions states that: (i) the Galaxy does not occupy a particular position, (ii) the Universe is homogeneous and isotropic. This statement does not agree with the recent astronomical observations in the range $\mathrm{z}$ lower than 0.05 which are in agreement with a cellular structure of the Universe. Here we present a local analysis of the inhomogeneity of the Universe. When $\mathrm{z}$ is greater than 0.05 our analysis cannot be applied because the astronomical sample of galaxies here processed is not complete. The two tools of the Poisson Voronoi tessellation (PVT) and the luminosity function for galaxies allow of building a new version of the local Cosmological Principle.

keywords

Cosmology: miscellaneous Cosmology: observations Cosmology: theory

\section{Introduction}

All the various theories which belong to cosmology are based on the "Cosmological Principle" which is now presented in two versions. The first version asserts that "There is nothing special about our location in the Universe," 
see Ryden (2003); Liddle \& Loveday (2009). The second version is "Viewed on a sufficiently large scale, the properties of the Universe are the same for all observers," see Keel (2007). Other versions which allow the formation of structures and incorporate probability distributions can be found in Schwarz (2009).

These two statements are strictly connected with the concept of isotropy and 3D homogeneity of the Universe. In the following we will show that

- The Local Super Cluster (LSC), the nearest part of the universe, has a dimension around 2 which is characteristic of a bi-dimensional space and therefore the concept of 3D homogeneity is violated by the observations.

- The concept of isotropy in the spatial distribution of galaxies is not confirmed due to the 3D cellular structure of galaxies.

In order to demonstrate the previous two statements this paper is organized as follows. Section 2 reviews the adopted cosmology. Section 3 reports a spherical cut of the 3D Voronoi diagram as well an introduction to the thick Voronoi faces. Section 4.2 reports a spherical cut of an astronomical catalog. Section 5 reports the most used luminosity function for galaxies, a modern version of the Malmquist bias, and the measure of the spatial dimensions of the LSC. Section 6 reports a revised version of the "Cosmological Principle."

\section{Elementary Cosmology}

Starting from Hubble (1929) the suggested correlation between expansion velocity and distance is

$$
V=H_{0} D=c z \quad,
$$

where the Hubble constant is $H_{0}=100 h \mathrm{~km} \mathrm{~s}^{-1} \mathrm{Mpc}^{-1}$, with $h=1$ when $h$ is not specified, $D$ is the distance in Mpc, $c$ is the velocity of light, and $z$ is the redshift. The redshift of galaxies is explained by the Doppler effect or by alternative theories such as the presence of a hot plasma with low density, see Brynjolfsson (2009). In a Euclidean, non-relativistic, and homogeneous universe, the flux of radiation, $f$, expressed in $\frac{L_{\odot}}{M p c^{2}}$ units, where $L_{\odot}$ represents 
the luminosity of the sun, is

$$
f=\frac{L}{4 \pi D^{2}}
$$

where $D$ is the distance of the galaxy expressed in Mpc, and

$$
D=\frac{c_{l} z}{H_{0}}
$$

\section{Voronoi Diagrams}

The points of a tessellation in 3D are of four types, depending on how many nearest neighbors in $E S$, the ensemble of seeds, they have. The name seeds derive from their role in generating cells. Basically we have two kinds of seeds, Poissonian and non Poissonian which generate the Poissonian Voronoi tessellation (PVT) and the non Poissonian Voronoi tessellation (NPVT). The Poissonian seeds are generated independently on the $X, Y$ and $Z$ axis in 3D through a subroutine which returns a pseudo-random real number taken from a uniform distribution between 0 and 1 . This is the case most studied and for practical purposes, the subroutine RAN2 was used, see Press et al. (1992). The non Poissonian seeds can be generated in an infinite number of different ways : some examples of NPVT are reported in Zaninetti (2009).

A point with exactly one nearest neighbor's is in the interior of a cell, a point with two nearest neighbors is on the face between two cells, a point with three nearest neighbors is on an edge shared by three cells, and a point with four neighbors is a vertex where three cells meet. The Abell clusters and therefore the galaxies were originally inserted on the Voronoi vertexes, which are the points which share the same distance from four seeds, see van de Weygaert \& Icke (1989). The target to reproduce in the previous analysis was the cluster-cluster correlation function. In order to explain the cellular structure of the universe the galaxies were later on collocated on the faces of a NPVT in order to reproduce the galaxy-galaxy correlation function and the cluster-cluster correlation function, see Zaninetti (1991). More detailed simulations of the various astronomical catalogs as well of the galaxy-galaxy correlation function were recently obtained inserting the galaxies on the faces of a PVT, see Zaninetti (2006, 2010).

According to this trend we will assume that the galaxies are situated on the faces of the PVT. Some of the properties of the PVT may be deduced 
from approximate arguments introducing the averaged radius of a polyhedron, $\bar{R}$ and the averaged diameter $\bar{D}=2 \bar{R}$. This theoretical quantity has its observational counterpart in the averaged diameter of the voids between galaxies, $\overline{D^{o b s}}$. A careful analysis of the effective radius of the voids between galaxies in SDSS DR7, see Section 4.1, derives $\overline{D^{o b s}}=\frac{36.46}{h} M p c$. The averaged volume is

$$
\bar{V}=\frac{4}{3} \pi \bar{R}^{3} \quad,
$$

and the averaged number of faces is $n$ which according to Okabe et al. (1992) is $n=15.35$.

The averaged surface area of a polyhedron, $\bar{S}$, is

$$
\bar{S}=4 \pi \bar{R}^{2},
$$

and the approximate area of a face, $\bar{A}$, is

$$
\bar{A}=\frac{4 \pi \bar{R}^{2}}{n} .
$$

The approximate side of a face, $\bar{l}$, is

$$
\bar{l}=\sqrt{\frac{4 \pi \bar{R}^{2}}{n}} .
$$

On assuming that our galaxy is at the center of an irregular face of a PVT, the galaxies on the other faces are comprised within a distance $D_{F}$

$$
D_{F} \approx \frac{\bar{l}}{2} .
$$

The cross sectional area of a PVT can also be visualized through a spherical cut characterized by a constant value of the distance from the center of the box, in this case expressed in $z$ units, see Figure 1 this intersection is called $V_{s}(2,3)$ where the index $s$ stands for sphere. The galaxies are thought to be situated on the faces of a PVT network and Figure 2 reports an example of a spherical cut.

An astronomical slice consists of a pie diagram with polar angle given by the right ascension and polar radius given by the redshift. An example of a slice of the Sloan Digital Sky Survey (SDSS) which contains all the galaxies 


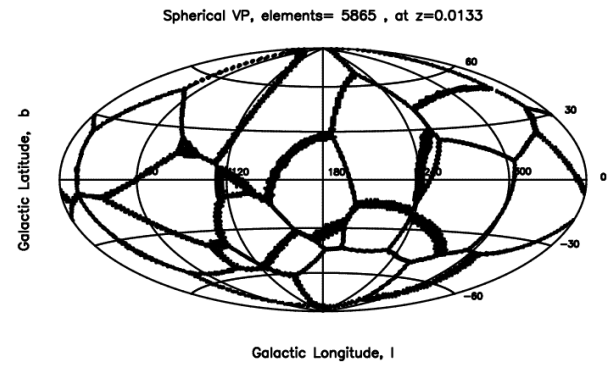

Figure 1: The Voronoi diagram $V_{s}(2,3)$ in the Hammer-Aitoff projection $0.0133 \leq z \leq 0.0144$ or $56.9 \mathrm{Mpc} \leq D \leq 61.67 \mathrm{Mpc}$ when $\bar{R}=18.16 \mathrm{Mpc}$.

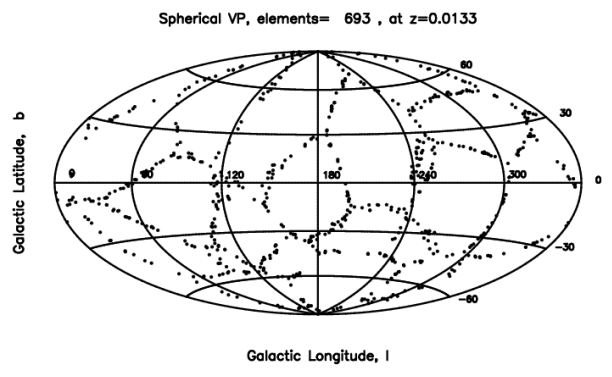

Figure 2: The Voronoi diagram $V_{s}(2,3)$ in the Hammer-Aitoff projection when 693 galaxies are extracted from the network of Figure 1 . 
within an opening angle in declination,$\varphi$, of $2.5^{\circ}$ around declination $=0$ can be found at http: //www.sdss3.org/gallery/sdss_pie2.php . Other surveys have the following opening angle in declination : $3^{\circ}$ for the second Center for Astrophysics (CFA2) survey, see Geller \& Huchra (1989) , $4^{\circ}$ for the 2dF Galaxy Redshift Survey (2dFGRS), see Colless et al. $(2001)$ and $4^{\circ}$ for the Six-degree Field instrument (6dF), see Jones et al. (2004). Particular attention should be paid to the fact that the astronomical slices are not a plane which intersects a Voronoi Network.

In order to quantify this effect we introduce a confusion distance, $D_{c}$, as the distance after which the half altitude of the slices equalizes the observed average diameter $\overline{D^{o b s}}$

$$
D_{c} \tan \left(\frac{\varphi}{2}\right)=\frac{1}{2} \overline{D^{o b s}},
$$

where $\varphi$ is the opening angle of the slice and $\overline{D^{o b s}}$ the averaged diameter of the voids. In the case of $\operatorname{SDSS} \varphi=2.5^{\circ}$ and therefore $D_{c}=835.4 / \mathrm{h} \mathrm{Mpc}$ when $\overline{D^{o b s}}=\frac{36.46}{h} \mathrm{Mpc}$. For distances greater than $D_{c}$ the voids in the distribution of galaxies are dominated by the confusion. For distances lower than $D_{c}$ the filaments of galaxies can be considered the intersection between a plane and the faces of the Voronoi Polyhedrons. The PVT offers a new classification for extragalactic aggregates.

1. Groups and clusters of galaxies are situated on the faces of the PVT with typical linear dimensions of $\approx 16 \mathrm{Mpc}$.

2. The local super-cluster is made by the network of many faces of the PVT. For distances equal to or slightly greater than $\overline{D^{o b s}} / 2$, a spherical cut should reveal $\approx 16$ voids .

3. The filaments of galaxies visible in the slices of oriented catalogs are due to the intersection between a plane and the PVT network of faces as first approximation. An improvement can be obtained by coding the intersection between the slice of a given opening angle and the PVT network of faces, see Zaninetti (2006, 2010). As an example Figure 3 reports both the CFA2 slice as well the simulated slice.

4. The Great Wall, see Geller \& Huchra (1989), is due to a combined effect of the photometric maximum, see Equation (19) and the PVT network of faces. 


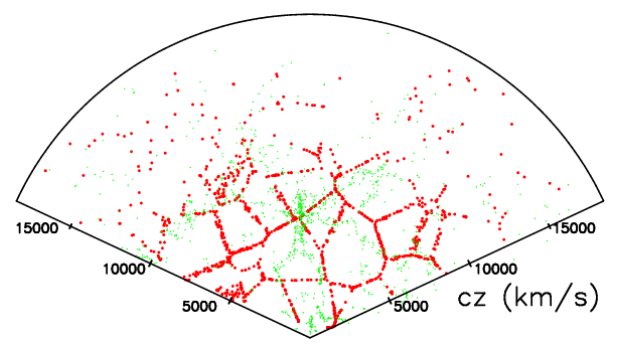

Figure 3: Polar plot of the real galaxies (green points) belonging to the second CFA2 redshift catalog and the simulated galaxies in the PVT framework (red points). More details can be found in Zaninetti (2006).

The cellular structure in the spatial distribution of galaxies can be generated in other approaches such as the long-range force acting only between non-baryonic particles, see Keselman et al. (2010).

\subsection{Thickness}

The concept of thickness of a face can be introduced by a numerical experiment. We generate $N_{r}$ random galaxies in $2 \mathrm{D}$ and then we progressively shift toward 3D increasing the thickness of the box from zero to a given value. The analysis of the number of galaxies, $N(R)$, as function of the distance can be done by assuming a power law dependence of the type

$$
N(R)=C R^{\beta}
$$

The two parameters $C$ and $\beta$ can be found from the following logarithmic transformation

$$
\ln (N(R))=\ln (C)+\beta \ln (R),
$$

which can be written as

$$
y=a_{L S}+b_{L S} x .
$$

The application of the least squares method through the FORTRAN subroutine LFIT from Press et al. (1992) allows of finding $a_{L S}, b_{L S}$ and the errors $\sigma_{a}$ and $\sigma_{b}$. The resulting dimension increases progressively with thickness, see Figure 4 . 


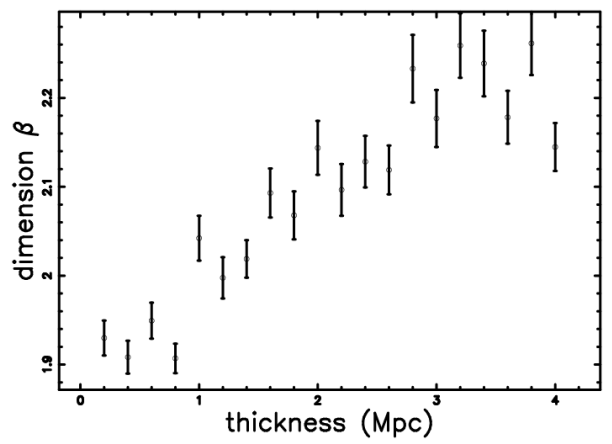

Figure 4: The dimension of the number of galaxies represented with vertical error bars as function of the thickness in Mpc.

The fact that the dimension is not exactly 2 at thickness $=0$, more exactly we have $\beta=1.8$ when $N_{r}=10000$, is due to the cardinality of $N_{r}$, as an example we have $\beta=1.96$ when $N_{r}=1000000$. This first analysis indicates that the dimension is function both of a geometrical factor as thickness and numerical factor, i.e. the number of considered galaxies. We now outline the method that allow us to insert the galaxies on the thick faces, see Zaninetti (1995, 2010). A practical implementation is to model a decreasing probability of having a galaxy in the direction perpendicular to the face. As an example we assume a probability, $p(x)$, of having a galaxy outside the face distributed as a Normal (Gaussian) distribution

$$
p(x)=\frac{1}{\sigma(2 \pi)^{1 / 2}} \exp -\frac{x^{2}}{2 \sigma^{2}},
$$

where $x$ is the distance in $M p c$ from the face and $\sigma$ the standard deviation in $M p c$. Once the complex 3D behavior of the faces of the Voronoi Polyhedron is set up we can memorize such a probability on a $3 \mathrm{D}$ grid $P(i, j, k)$ which can be found in the following way

- In each lattice point $(i, j, k)$ we search for the nearest element belonging to a Voronoi face. The probability of having a galaxy is therefore computed according to formula (13).

- A number of galaxies, $N_{G}=n_{*} \times$ side ${ }^{3}$ is then inserted in the box; here $n_{*}$ represents the density of galaxies 


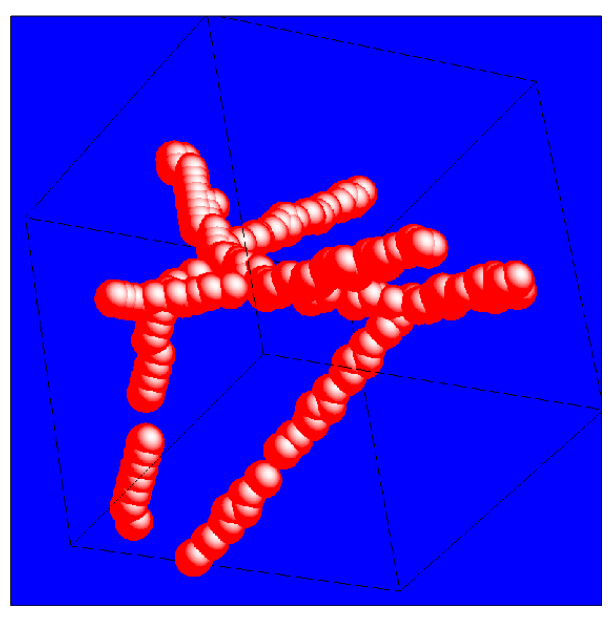

Figure 5: 3D visualization of the edges of the Poissonian Voronoi-diagram. The parameters are pixels $=60, N_{s}=60$, side $=89 \mathrm{Mpc}, h=0.7$ and amplify $=1.2$.

Figure 5 visualizes the edges belonging to the Voronoi diagrams and Figure 6 represents a cut in the middle of the probability, $P(i, j, k)$, of having a galaxy to a given distance from a face.

We are now ready to evaluate the spatial dimension in two cases

- ntrials centers randomly situated on a face ,

- ntrials centers randomly situated on a edge,

see Table 1. The case of evaluation starting from the edges produces a bigger dimension because we enclose three faces rather than one.

\section{Observations}

This section processes the Sloan Digital Sky Survey Data Release 7 (SDSS DR7) and the Two-Micron All Sky Survey (2MASS). 


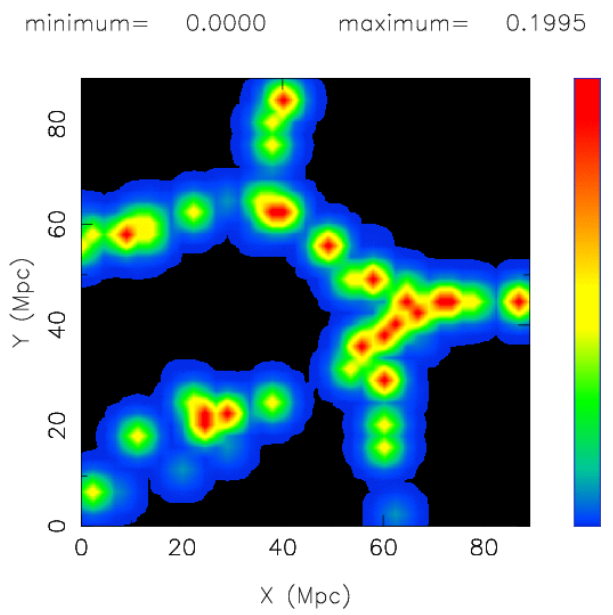

Figure 6: Cut in the middle of the $3 \mathrm{D}$ grid $P(i, j, k)$ which represents a theoretical 2D map of the probability of having a galaxy. The Voronoi parameters are the same as in Figure 5 and $\sigma=0.8 M p c$. The $\mathrm{X}$ and $\mathrm{Y}$ units are in Mpc.

Table 1: The dimension in two cases when ntrial $=10$.

\begin{tabular}{lc}
\hline \hline case & value \\
faces & $1.65 \pm 0.1$ \\
edges & $1.79 \pm 0.07$
\end{tabular}


Table 2: The statistical parameters of the effective radius in SDSS DR7.

\begin{tabular}{lc}
\hline \hline parameter & value \\
mean & $18.23 h^{-1} M p c$ \\
variance & $23.32 h^{-2} M p c^{2}$ \\
standard deviation & $4.82 h^{-1} M p c$ \\
kurtosis & 0.038 \\
skewness & 0.51 \\
maximum value & $34.12 h^{-1} \mathrm{Mpc}$ \\
minimum value & $9.9 h^{-1} \mathrm{Mpc}$ \\
\hline \hline
\end{tabular}

\subsection{Observed Statistics of the Voids}

The distribution of the effective radius between the galaxies of SDSS DR7 has been reported in Pan et al. (2012) and is also possible to extract the catalog which is visible at

http://www.physics.drexel. edu/ pan/voidcatalog.html

; this catalog contains 1054 voids and Table 2 reports their basic statistical parameters.

\subsection{The 2MASS}

The 2MASS is a catalog of galaxies which has instruments in the near-infrared $\mathrm{J}, \mathrm{H}$ and $\mathrm{K}$-bands $(1-2.2 \mu \mathrm{m})$ and therefore detects the galaxies in the so called "Zone of Avoidance," see Jarrett (2004); Crook et al. (2007). At the moment of writing the 2MASS Redshift Survey (2MRS) consists of 44599 galaxies with redshift in the interval $0 \leq z \leq 0.09$, see Huchra et al. (2012).

Figure 7 reports a spherical cut at a given radius in $z$ of the LSC according to 2MRS, which is available online at https://www.cfa.harvard.edu/ huchra/seminar/lsc/lsc.dat. The LSC is centred on the Virgo cluster, has a flattened structure and it's radius is $\approx 40$ Mpc. 


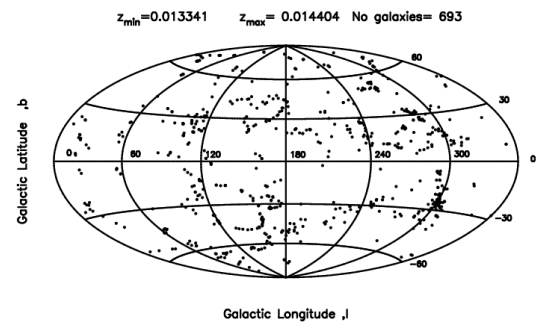

Figure 7: Hammer-Aitoff projection in galactic coordinates of a spherical cut of the LSC data at $0.0133 \leq z \leq 0.0144$ or $56.9 M p c \leq D \leq 61.67$.

A way to smooth the results of Figure 7 is through contour of isodensity of galaxies in a cube of sides equal to $16 \mathrm{Mpc}$ at a given distance, see Figure 8 . The previous figure contains voids in which the density of galaxies is zero. In order to have in all regions of the spherical cut a minimum density of 1 galaxy/(chosen volume) we consider the density as evaluated in a cube which has a side equal the averaged diameter as given by SDSS DR7, see Figure 9 . Now the density of galaxies has a minimum value of 1 at the center of a void and a maximum value of 270 on a side which comes out from the intersection of a sphere with a PVT. This means that the density of galaxies varies over more than two decades on a spherical cut.

The concept of homogeneity can be associated to a random sample which has the same cardinality of the astronomical galaxies but placed at random in the same analyzed shell; Figure 10 reports an example of such random galaxies. The ratio

$$
H(r)=\frac{N_{G}(r)}{\left\langle N_{R}(r)\right\rangle}
$$

quantifies the homogeneity of the astronomical sample with $N_{G}(r)$ representing the number of real galaxies and $N_{R}(r)$ the random galaxies in a cube of side $2 r,\left\langle N_{R}(r)\right\rangle$ represents the number of random galaxies averaged over $91 \times 181$ directions. A value of of $H=1$ means that the sample is homogeneous, a value lower that 1 means that the sample is not homogeneous. 
Figure 8: Logarithmic contours of density of galaxies of the Large Super Cluster. The density is evaluated in cubes of side $=16 \mathrm{Mpc}$ at $z=0.0133$ or $D=56.9 M p c$.

Figure 9: Linear contours of density of galaxies of the Large Super Cluster. The density is evaluated in cubes of side $=16 \mathrm{Mpc}$ at $z=0.0133$ or $D=$ $56.9 M p c$. 


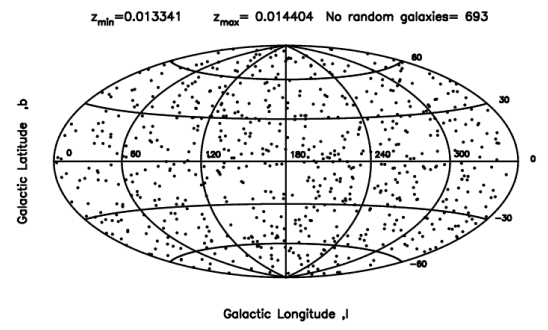

Figure 10: Hammer-Aitoff projection in galactic coordinates of a spherical cut of a random sample of galaxies at $0.0133 \leq z \leq 0.0144$ or $56.9 M p c \leq$ $D \leq 61.67$. The number of galaxies is the same of Figure 7 .

An example of the various value of $H$ is reported in Figure 10 and Figure 12 reports the numerical survival function for the grid points. From this last figure is possible to conclude that we have non homogeneity over the $68 \%$ of the grid points of the spherical cut.

Figure 9 which shows the linear density contours of galaxies and Figure 11 which shows the linear contours of $\mathrm{H}$ are similar because the numbers of simulated galaxies in the selected cube of the random sample fluctuates less in respect to the sample of galaxies.

\section{$5 \quad$ Luminosity effects}

This section reports the luminosity function for galaxies, the Malmquist bias which allows to build a complete sample of galaxies, and the numerical determination of the spatial dimension of the LSC.

\subsection{Luminosity Function}

A model for the luminosity of galaxies is the Schechter function

$$
\Phi(L) d L=\left(\frac{\Phi^{*}}{L^{*}}\right)\left(\frac{L}{L^{*}}\right)^{\alpha} \exp \left(-\frac{L}{L^{*}}\right) d L
$$




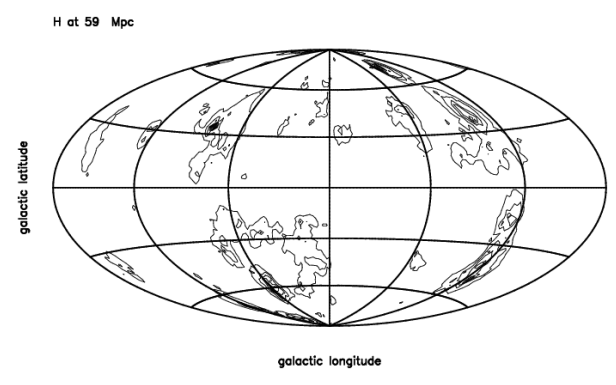

Figure 11: Linear contours of $\mathrm{H}$ as given by (14) in the Large Super Cluster. The density of the astronomical and random samples are evaluated in cubes of side $=16 \mathrm{Mpc}$ at $z=0.0133$ or $D=56.9 \mathrm{Mpc}$.

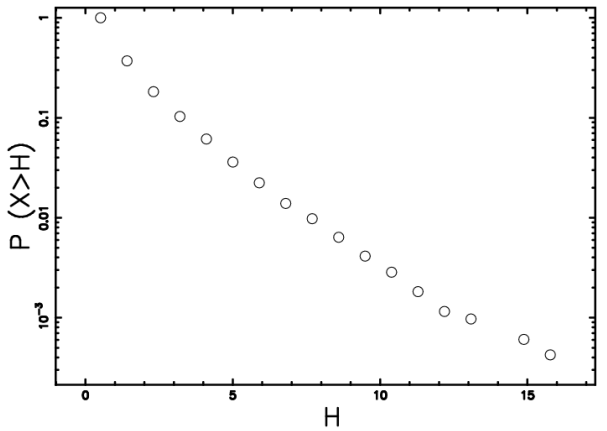

Figure 12: Survival function of of the ensemble of $91 \times 181$ grid points as function of $H$ at $D=56.9 \mathrm{Mpc}$. The median is at $H=0.6$, in an homogeneous sample it should be at $H=1$. 
Table 3: The parameters of the Schechter function and bolometric magnitude for the 2MRS in the $K_{s}-$ band.

\begin{tabular}{lc}
\hline \hline parameter & $2 M R S$ \\
$M^{*}-5 \log _{10} h[$ mags $]$ & $(-23.44 \pm 0.03)$ \\
$\alpha$ & $-0.96 \pm 0.05$ \\
$\Phi^{*}\left[h^{3} M p c^{-3}\right]$ & $\left((1.08 \pm 0.16) 10^{-2}\right)$ \\
$M_{K_{S}}^{\odot}$ & 3.39 \\
$h$ & 0.7 \\
\hline \hline
\end{tabular}

where $\alpha$ sets the slope for low values of $L, L^{*}$ is the characteristic luminosity, and $\Phi^{*}$ is a normalization. This function was suggested by Schechter (1976) and the distribution in absolute magnitude is

$$
\begin{aligned}
\Phi(M) d M=\quad & (0.4 \ln 10) \Phi^{*} 10^{0.4(\alpha+1)\left(M^{*}-M\right)} \\
& \times \exp \left(-10^{0.4\left(M^{*}-M\right)}\right) d M
\end{aligned}
$$

where $M^{*}$ is the characteristic magnitude as derived from the data. The parameters of the Schechter function concerning the $2 \mathrm{MRS}$ can be found in Cole et al. (2001) and are reported in Table 3.

The number of galaxies at a given flux $f$ as a function of the redshift $z$, see Formula (1.104) in Padmanabhan (1996), is

$$
\frac{d N}{d \Omega d z d f}=4 \pi\left(\frac{c}{H_{0}}\right)^{5} z^{4} \Phi\left(\frac{z^{2}}{z_{c r i t}^{2}}\right)
$$

where $d \Omega, d z$, and $d f$ are the differentials of the solid angle, the red-shift, and the flux, respectively, and

$$
z_{c r i t}^{2}=\frac{H_{0}^{2} L^{*}}{4 \pi f c^{2}}
$$

where $c$ is the velocity of light. The number of galaxies at a given flux has a maximum at $z=z_{\max }$, where

$$
z_{\max }=z_{\text {crit }} \sqrt{\alpha+2}
$$




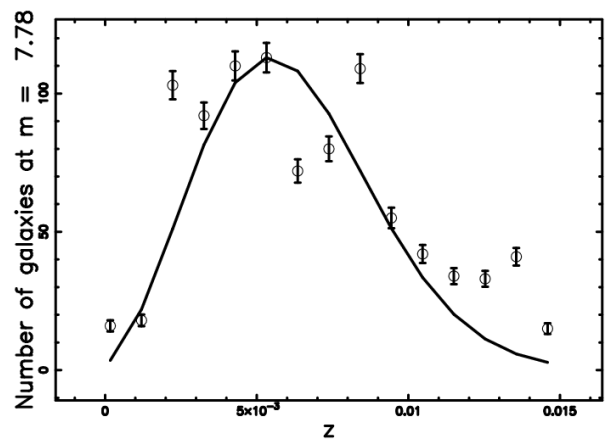

Figure 13: The galaxies of the 2MRS with $7.23 \leq m \leq 8.92$ or $4881116 \frac{L_{\odot}}{M p c^{2}} \leq f \leq 23044324 \frac{L_{\odot}}{M p c^{2}}$ are organized in frequencies versus heliocentric redshift, (empty circles); the error bar is given by the square root of the frequency. The maximum frequency of observed galaxies is at $z=0.0058$. The full line is the theoretical curve generated by (17), the Schechter function of luminosity, with parameters as in Table 3. In this plot $\mathcal{M}_{\odot}=3.39, h=$ $0.7, \chi^{2}=132.93$ and the number of bins 15 .

Figure 13 reports the number of observed galaxies in the 2MRS catalog at a given apparent magnitude and the theoretical curve as represented by Equation (17). The merit function $\chi^{2}$ can be computed as

$$
\chi^{2}=\sum_{j=1}^{n}\left(\frac{n_{\text {theo }}(z)-n_{\text {astr }}(z)}{\sigma_{n_{\text {astr }}(z)}}\right)^{2},
$$

where $n$ is number of data, the two indexes theo and astr stand for theoretical and astronomical, respectively and $\sigma_{n_{a s t r}(z)}{ }^{2}$ is the variance of the astronomical number of data; the obtained value is reported in the caption of Figure 13.

On analyzing Figure 13 we suggest that the disagreement between theory and observations is due to the fact that the number of galaxies does not increases exactly with $R^{3}$ as inserted in the theory but with $R^{1.87}$, see equation (26), as given by the cellular structure. 


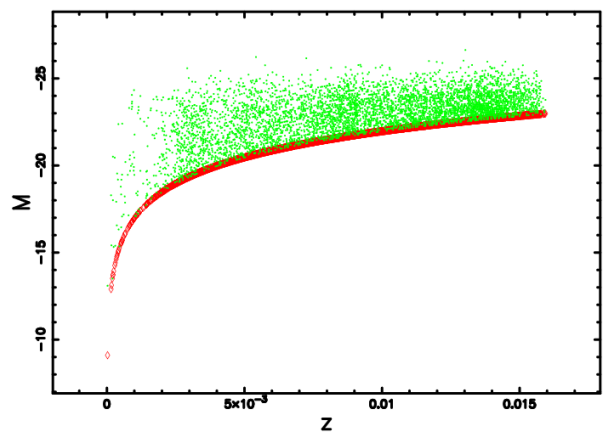

Figure 14: The absolute magnitude $M$ of 5512 galaxies belonging to the 2MRS when $\mathcal{M}_{\odot}=3.39$ and $H_{0}=70 \mathrm{~km} \mathrm{~s}^{-1} \mathrm{Mpc}^{-1}$ (green points). The upper theoretical curve as represented by Equation (21) is reported as the red thick line when $m_{L}=11.19$.

\subsection{Malmquist Bias}

The Malmquist bias, see Malmquist (1920, 1922), was originally applied to the stars and later on to the galaxies by Behr (1951). The observable absolute magnitude as a function of the limiting apparent magnitude, $m_{L}$, is

$$
M_{L}=m_{L}-5 \log _{10}\left(\frac{c z}{H_{0}}\right)-25
$$

The previous formula predicts, from a theoretical point of view, an upper limit on the maximum absolute magnitude which can be observed in a catalog of galaxies characterized by a given limiting magnitude and Figure 14 reports such a curve and the galaxies of the $2 \mathrm{MRS}$.

The interval covered by the LF of galaxies, $\Delta M$, is defined by

$$
\Delta M=M_{\max }-M_{\min }
$$

where $M_{\max }$ and $M_{\min }$ are the maximum and minimum absolute magnitude of the LF for the considered catalog. The real observable interval in absolute magnitude, $\Delta M_{L}$, is

$$
\Delta M_{L}=M_{L}-M_{\min }
$$


We can therefore introduce the range of observable absolute maximum magnitude expressed in percent, $\epsilon_{s}(z)$, as

$$
\epsilon_{s}(z)=\frac{\Delta M_{L}}{\Delta M} \times 100 \%
$$

This is a number which represents the completeness of the sample and, given the fact that the limiting magnitude of the $2 \mathrm{MRS}$ is $m_{L}=11.19$, it is possible to conclude that the 2MRS is complete for $z \leq 0.00016$. This efficiency expressed as a percentage can be considered a version of the Malmquist bias.

The previous treatment does not take into account the $K$-corrections between observed $(Q)$ and desired $(R)$ bandpasses, $K_{Q R}(z)$, see equation (1) Blanton \& Roweis (2007). The $K$-correction depends from the chosen catalog and bandpass. In our case we are interested in band $K_{s}$ in the 2MASS and the following approximate relationship can be drawn from Figure 14 in Blanton \& Roweis (2007):

$$
K_{K}(z) \approx 1.2 z
$$

As an example at $z \approx 0.016$, which is the maximum processed value of redshift of the LSC, the $K$-corrections are $0.02 \mathrm{mag}$. This means that the $K$-corrections are negligible when applied to the LSC, which is a subset of the 2MASS.

\subsection{The Radial Distribution of Galaxies}

In a 3D random distribution, the number of galaxies grows $\propto R^{3}$ where $R$ is the distance from the center (our galaxy) in Mpc. In a framework of galaxies situated on the first face of a PVT their number should grow $\propto R^{2}$ assuming that $R$ denotes the distance from the center of the face. The counting of the nearest galaxies can be done in light of the Malmquist bias and the first face of a PVT. According to (8), the galaxies on the first face will terminate at $D_{F} \approx 8.75 M p c$ which corresponds to $z=0.00204$. The Malmquist line as represented by (21) suggests that the sample of galaxies in the interval $0<z<0.00204$ and characterized $M<-18.51$ is complete. According to equation 10 the spatial behavior is

$$
\begin{gathered}
N(R)=(3.52 \pm 0.49) R^{(1.87 \pm 0.09)} \\
\text { when } 0<z<0.00204 \text { and } M<-18.51,
\end{gathered}
$$




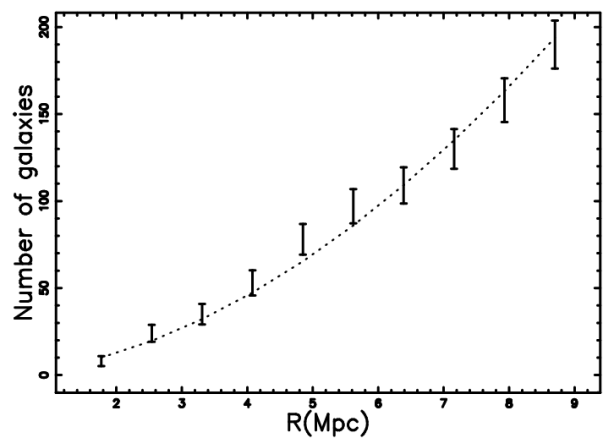

Figure 15: The number of galaxies as function of the distance in Mpc for the $2 \mathrm{MRS}$ catalog (full line) and observed number of galaxies with vertical error bars. The error is computed as the square root of the number of galaxies in the considered bin.

and Figure 15 reports such a relationship.

This last result can be tested on a random 2D sample in which the galaxies are generated on a plane having the same area of the astronomical analyzed region. The result of the simulation for the random galaxies gives

$$
\begin{gathered}
N(R)=(1.43 \pm 0.03) R^{(2.03 \pm 0.082)} \\
\text { when } 0<z<0.00204 .
\end{gathered}
$$

A comparison of the two curves of growth ( random galaxies and observed galaxies) is reported in Figure 16.

Others approaches use the generalized fractal dimension estimator to (i) analyze the scaling properties of the clusters of galaxies, see Borgani et al. (1993), (ii) determine the scale of homogeneity in the Universe as derived from various galaxy redshift surveys, see Bagla et al. (2008), (iii) study the transition to homogeneity, defined as the scale above which the fractal dimension of the underlying galaxies distribution is equal to the ambient dimension of the space in which the galaxies are distributed, see Yadav et al. (2010). 


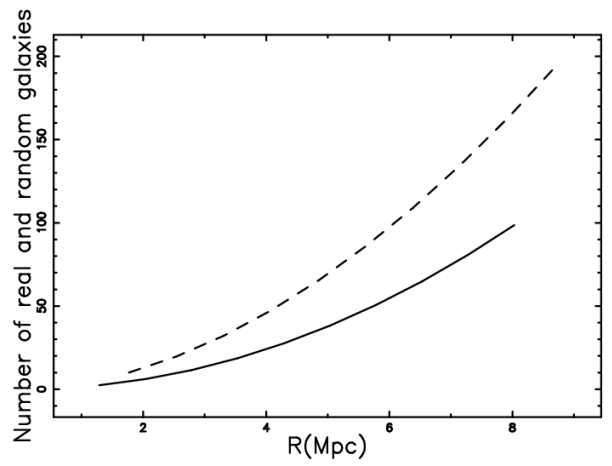

Figure 16: The number of real galaxies as function of the distance in Mpc for the 2MRS catalog (full line) and number of random galaxies generated on a plane (dashed line).

\section{Conclusions}

In Section 4.2 we learned that the concept of homogeneity does not correspond to the observations of LSC because the $63 \%$ of $91 \times 181$ grid points processed at a distance of $\approx 59 \mathrm{Mpc}$ have the indicator of homogeneity lower than 1. The concept of a non-isotropic universe is a consequence of the previous statement, in other words, the appearance of the universe depends on the chosen observer/galaxy. These facts, based on the theoretical and observational bases, allow us to assert a local new version of the Cosmological Principle "The galaxies, as well our galaxy, are situated on the faces of a Voronoi polyhedron. The spatial distribution of galaxies will follow approximately the geometrical rules as well the standard photometric rules." A first set of consequences can be drawn

1. The number of galaxies in the first face of a PVT should grown as $N(R) \propto R^{2}$. An analysis of the number of galaxies in the first 8.75 Mpc of the LSC gives $N(R) \propto R^{1.87}$.

2. A new definition of the density of galaxies on the faces of a PVT, $n_{f}$, which in the framework of thick faces is

$$
n_{f} \approx n \frac{1}{3 \rho} \text {, }
$$


where $\rho=s / \bar{R}$ is a dimensional parameter lower than $1, s$ is the thickness of the faces, and $n$ is the usual density of galaxies.

3. The so called "filaments of galaxies" are here classified as 2D sections of a 3D PVT which are made by irregular polygons.

4. The reformulation here presented is in agreement with the anisotropies in the short and intermediate gamma ray bursts which are explained by a breakdown of the Cosmological Principle, see Meszaros et al. (2009) Mészáros et al. (2009).

5. The theoretical dimension which regulates the number of galaxies as function of the distance is different when the center of the selected sphere belongs to a thick face or a thick edge.

Different approaches which analyze the SDSS seem to suggest the validity of cosmological principle and they are: (i) the test the homogeneity of the universe at $\mathrm{z} \approx 0.3$ with the luminous red galaxy (LRG) spectroscopic sample of the SDSS, see Hogg et al. (2005), (ii) a multifractal analysis of the galaxy distribution in a volume-limited sub-sample from the (SDSS) which finds that the galaxy distribution becomes homogeneous at a length-scale between 60/h Mpc and 70/h Mpc, see Sarkar et al. (2009). We remember that 70/h Mpc as a linear dimension comprises $\approx 2$ PVT cells. The analysis here presented is limited to galaxies within $8 \mathrm{Mpc}$ in order to measure the spatial dimension. Tests carried out by other authors also do not find homogeneity within 8 Mpc. Rather, the transition to homogeneity is found to occur at a much larger length-scale. So there does not appear to be any inconsistency with the findings of our paper and the currently accepted picture of the transition to homogeneity. In the framework of the cellular universe the transition from non homogeneous to homogeneous regime is reached when the cube in which the density of galaxies is evaluated comprises many cells.

\section{References}

Bagla, J. S., Yadav, J., \& Seshadri, T. R. 2008, MNRAS , 390, 829

Behr, A. 1951, Astronomische Nachrichten, 279, 97 
Blanton, M. R., \& Roweis, S. 2007, AJ , 133, 734

Borgani, S., Murante, G., Provenzale, A., \& Valdarnini, R. 1993, Phys. Rev. E , 47, 3879

Brynjolfsson, A. 2009, in Astronomical Society of the Pacific Conference Series, Vol. 413, Astronomical Society of the Pacific Conference Series, ed. F. Potter, 169-189

Cole, S., Norberg, P., Baugh, C. M., Frenk, C. S., Bland-Hawthorn, J., Bridges, T., Cannon, R., Colless, M., Collins, C., Couch, W., Cross, N., Dalton, G., De Propris, R., Driver, S. P., Efstathiou, G., Ellis, R. S., Glazebrook, K., Jackson, C., Lahav, O., Lewis, I., Lumsden, S., Maddox, S., Madgwick, D., Peacock, J. A., Peterson, B. A., Sutherland, W., \& Taylor, K. 2001, MNRAS , 326, 255

Colless, M., Dalton, G., Maddox, S., \& et al. 2001, MNRAS , 328, 1039

Crook, A. C., Huchra, J. P., Martimbeau, N., Masters, K. L., Jarrett, T., \& Macri, L. M. 2007, ApJ , 655, 790

Geller, M. J., \& Huchra, J. P. 1989, Science, 246, 897

Hogg, D. W., Eisenstein, D. J., Blanton, M. R., Bahcall, N. A., Brinkmann, J., Gunn, J. E., \& Schneider, D. P. 2005, ApJ , 624, 54

Hubble, E. 1929, Proceedings of the National Academy of Science, 15, 168

Huchra, J. P., Macri, L. M., Masters, K. L., \& et al. 2012, ApJS , 199, 26

Jarrett, T. 2004, PASA , 21, 396

Jones, D. H., Saunders, W., Colless, M., Read, M. A., \& Parker, Q. A. e. 2004, MNRAS , 355, 747

Keel, W. C. 2007, The road to galaxy formation (Berlin: Springer)

Keselman, J. A., Nusser, A., \& Peebles, P. J. E. 2010, Phys. Rev. D , 81, 063521

Liddle, A., \& Loveday, J. 2009, The Oxford Companion to Cosmology (Oxford: Oxford University Press) 
Malmquist , K. 1920, Lund Medd. Ser. II, 22, 1

—. 1922, Lund Medd. Ser. I, 100, 1

Meszaros, A., Balazs, L. G., Bagoly, Z., \& Veres, P. 2009, in American Institute of Physics Conference Series, Vol. 1133, American Institute of Physics Conference Series, ed. C. Meegan, C. Kouveliotou, \& N. Gehrels, 483-485

Mészáros, A., Balázs, L. G., Bagoly, Z., \& Veres, P. 2009, Baltic Astronomy, 18,293

Okabe, A., Boots, B., \& Sugihara, K. 1992, Spatial tessellations. Concepts and Applications of Voronoi diagrams (Chichester, New York: Wiley)

Padmanabhan, T. 1996, Cosmology and Astrophysics through Problems (Cambridge: Cambridge University Press)

Pan, D. C., Vogeley, M. S., Hoyle, F., Choi, Y.-Y., \& Park, C. 2012, MNRAS , 421, 926

Press, W. H., Teukolsky, S. A., Vetterling, W. T., \& Flannery, B. P. 1992, Numerical Recipes in FORTRAN. The Art of Scientific Computing (Cambridge: Cambridge University Press)

Ryden, B. 2003, Introduction to Cosmology (San Francisco, CA, USA: Addison Wesley)

Sarkar, P., Yadav, J., Pandey, B., \& Bharadwaj, S. 2009, MNRAS , 399, L128

Schechter, P. 1976, ApJ , 203, 297

Schwarz, D. J. 2009, eprint arXiv:0905.0384

van de Weygaert, R., \& Icke, V. 1989, A\&A , 213, 1

Yadav, J. K., Bagla, J. S., \& Khandai, N. 2010, MNRAS , 405, 2009

Zaninetti, L. 1991, A\&A , 246, 291

-. 1995, A\&AS , 109, 71 
—. 2006, Chinese J. Astron. Astrophys. , 6, 387

—. 2009, Phys. Lett. A , 373, 3223

—. 2010, Revista Mexicana de Astronomia y Astrofisica, 46, 115 